\title{
Nutrición al final de la vida: perspectiva paliativa del cirujano
}

\author{
Nutrition at the-end-of-life: Palliative perspective of the surgeon \\ Nutrição no final da vida: A perspectiva paliativa do cirurgião
}

\author{
Juan Carlos Sabogal Olarte ${ }^{1 *}$, Carmen Santos García', Ana Cristina Ortiz ${ }^{2}$, \\ Carlos Eduardo Rey Chaves' ${ }^{1}$ Danny Conde Monroy'
}

Recibido: 20 de octubre de 2020. Aceptado para publicación: 10 de diciembre de 2020.

https://doi.org/10.35454/rncm.v4n2.220

\begin{abstract}
Resumen
Introducción: el manejo médico en el final de la vida en pacientes con cáncer representa un reto clínico y un dilema ético y moral. No resulta fácil establecer las estrategias de acompañamiento para el paciente y su familia, ni establecer las estrategias nutricionales a seguir. El objetivo de esta revisión es analizar los aspectos teóricos establecidos en la literatura sobre la definición de final de la vida y las estrategias para el manejo nutricional en pacientes con cáncer desde la experiencia del Servicio de Cirugía Hepatobiliar y Pancreática del Hospital Méderi en Bogotá, Colombia.
\end{abstract}

Métodos: se realizó una búsqueda de la literatura con los términos "nutrición", "cuidado paliativo", "nutrición en cáncer", "final de la vida". Se describen las diferentes estrategias de manejo nutricional para los pacientes con patología oncológica hepatobiliar y pancreática que se encuentran en la etapa de final de vida.

Resultados: el deterioro clínico del paciente en esta etapa de vida es irreversible, y los aportes proteico-calóricos a través del suministro parenteral o enteral no cambian el pronóstico del paciente. El objetivo final de la terapia nutricional en el paciente en el final de vida es principalmente la calidad de vida. La apropiada comunicación con el paciente y la familia es fundamental para disminuir la ansiedad y facilitar la toma de decisiones.

\section{Summary}

Introduction: The care of cancer patients at the end-of-life represents a clinical challenge and an ethical and moral dilemma. Establishing follow-up strategies for the patient and his or her family, or the nutritional strategies to undertake is not easy. The objective of this review is to analyze theoretical aspects defined in the literature on the definition of endof-life and the nutritional management strategies for cancer patients obtained from the experience of the Hepatobiliary and Pancreatic Surgery Service at Méderi Hospital in Bogotá, Colombia.

Methods: A literature search was carried out using the terms: "nutrition", "palliative care", "nutrition in cancer", "end-of-life". The different end-of-life nutritional strategies for patients with hepatobiliary and pancreatic terminal disease are described.

Results: Clinical deterioration of patients in this stage of life is irreversible, and the protein and energy intake delivered through a parenteral or enteral access, do not change the patient's prognosis. The ultimate goal of nutritional therapy in the patient at the end-of-life is maintaining quality of life. Proper communication with the patient and his or her family is essential to reduce anxiety and facilitate decision-making.

Conclusion: Nutritional management in cancer patients at the end-of-life is a huge medical challenge. The experience of

\section{Resumo}

Introdução: O manejo médico do paciente no final da vida em pacientes com câncer representa um desafio clínico e um dilema ético e moral. Não é fácil estabelecer as estratégias de acompanhamento para o paciente e sua familia, assim como as estratégias nutricionais a serem seguidas. O objetivo desta revisão é analisar os aspectos teóricos estabelecidos na literatura sobre a definição de final da vida e as estratégias de manejo nutricional, em pacientes com câncer a partir da experiência do Serviço de Cirurgia Hepatobiliar e Pancreática do Hospital Mederi de Bogotá, Colômbia.

Métodos: foi realizada uma pesquisa bibliográfica, com os termos "nutrição", "cuidado paliativo", "nutrição em câncer", "final da vida". São descritas as diferentes estratégias de manejo nutricional para pacientes com patologia oncológica hepatobiliar e pancreática que se encontram na fase de final da vida.

Resultados: a deterioração clínica do paciente nesta fase é irreversível e as contribuições protéico-calóricas por via parenteral ou enteral não alteram o prognóstico do paciente. O objetivo final da terapia nutricional no paciente nesta etapa é principalmente a melhora da qualidade de vida. A comunicação adequada com o paciente e a família é essencial para reduzir a ansiedade e facilitar a tomada de 
Conclusión: el manejo nutricional de los pacientes oncológicos al final de la vida es un enorme reto médico. La experiencia del servicio HPB aporta una perspectiva humana y paliativa, enfocada en el acompañamiento del paciente y su familia, recuperando el concepto fundamental de la relación médico-paciente.

Palabras clave: nutrición, cuidado paliativo, cáncer de páncreas, calidad de vida. the Hepatobiliary and Pancreatic Surgery Service provides a human and palliative perspective focused on accompanying the patient and his or her family, thus recovering the fundamental concept of the doctor-patient relationship.

Keywords: Nutrition; Palliative Care; Pancreatic Cancer; Quality of Life. decisões.

Conclusão: $O$ manejo nutricional dos pacientes oncológicos no final da vida é um grande desafio médico. A vivência do serviço HPB proporciona uma perspectiva humanizada e paliativa voltada para o acompanhamento do paciente e sua família, resgatando o conceito fundamental da relação médico-paciente.

Palavras-chave: nutrição, cuidados paliativos, câncer de pâncreas, qualidade de vida.
' Servicio de Cirugía Hepatobiliar y Pancreática, Hospital Universitario Mayor Méderi, Bogotá DC, Colombia.

*Correspondencia: Juan Carlos Sabogal Olarte. juan.sabogal@mederi.com.co

\section{INTRODUCCIÓN}

El final de la vida es un concepto que tiene diferentes aristas, y que involucra la ética, la moral, la religión, los cuidados paliativos, la cirugía, la medicina crítica, la psicología, la nutrición y la psiquiatría, entre otros ${ }^{(1)}$. Por tanto, esta variedad de perspectivas hace su concepto controversial y complejo de definir. Pretender tener las respuestas claras sobre qué es lo mejor y apropiado, o lo que debería ser el manejo del paciente en el final de la vida, es casi una ilusión. Nadie puede afirmar con vehemencia que la mejor manera de enfocar a un paciente en el final de la vida es la eutanasia o, por el contrario, establecer medidas heroicas para prolongarla. Las creencias religiosas, las tradiciones y la concepción misma de la vida son tan personales que generalizar en este sentido, más que utópico, resulta irrespetuoso y muy pretensioso.

Cuando nos enfrentamos a pacientes con enfermedades intratables o incurables, desde una perspectiva alopática, no significa necesariamente que no sean manejables desde una mirada humana. Este artículo, escrito por el grupo de Cirugía Hepatobiliar y Pancreática (HPB) del Hospital Méderi en Bogotá, Colombia, no solo plasma la revisión de la literatura sobre el manejo nutricional en los pacientes al final de la vida, sino que comparte la experiencia y perspectiva de este problema, que a diario enfrentamos en los pacientes que manejamos en nuestro servicio.
Claramente, la experiencia en HPB no nos hace expertos en cuidados paliativos, pero sí nos permite compartir la firme convicción de que el compromiso del cirujano tiene que ir más allá de un procedimiento quirúrgico y penetrar las fibras del paciente, la familia y del cirujano, que antes de operar es un ser humano que entrega su labor y su alma en cada paciente. Es por esto por lo que el servicio HPB del Hospital Méderi se ha enfocado no solo en el manejo quirúrgico de los pacientes con cáncer de hígado, vías biliares y páncreas, sino que se encarga del enfoque médico y paliativo de los pacientes que no son candidatos a cirugía por el estado avanzado de la enfermedad o por la condición misma del paciente.

Estamos convencidos de la responsabilidad ética y moral del médico tratante, en este caso, del grupo $\mathrm{HPB}$, con los pacientes y familiares, en establecer una cercana relación, y una honesta y cálida comunicación. De igual manera, hay una clara obligación como educadores, de enseñar a todo el grupo de estudiantes de pregrado y posgrado, la importancia de establecer un fuerte vínculo entre el grupo médico y el paciente, que no se puede limitar a informar de un procedimiento quirúrgico, sino del acompañamiento permanente en todo el proceso de enfermedad, puesto que no se puede definir con certeza si se alcanzará el objetivo de curar el cáncer o no, o si el desenlace va a ser mortal por la naturaleza misma de la enfermedad. La perspectiva paliativa del cirujano es fundamental porque es el experto en 
la enfermedad y en las posibles soluciones curativas y paliativas de la patología, por ende, su obligación está en brindar el acompañamiento permanente al paciente para ofrecer la mejor calidad de la atención, aun cuando no haya opciones curativas de la enfermedad.

En este contexto, el final de la vida y el paciente terminal son términos que han venido aclarándose por los expertos en cuidados paliativos, y que definiremos más adelante. De igual manera, resulta complejo establecer las metas nutricionales para un paciente en estas condiciones, especialmente en el final de la vida, tema que es el objeto de este escrito. Es importante mencionar que hablar de nutrición en estos pacientes no solo es un desafío para el médico tratante y el paciente, sino una situación compleja de manejar con la familia, quienes son los que deben enfrentar el declive de un ser querido con las manos cruzadas y sin alternativas.

A pesar de que la medicina moderna ha desarrollado medicamentos, vacunas, cirugías, quimioterapia, entre otros, que ha permitido prolongar y mejorar la calidad de vida de los pacientes (hoy en día, la expectativa de vida en Colombia está cerca de los 77 años), siempre hay un final de la vida, y esto es inevitable. Es por esto por lo que las intervenciones que realizamos durante este tiempo continúan siendo un tema de debate, en el cual cada vez hay más claridad teórica, científica y con mayor concientización, establecida por organizaciones, sobre el derecho a morir dignamente y el crecimiento de los cuidados paliativos entre otras. Afrontar el final de vida no ha hecho parte del paradigma en el que vivimos en la época moderna. Este se enfoca en tener más experiencias y en vivir la vida al máximo, y rara vez en cómo afrontar la experiencia del proceso de muerte.

La nutrición del paciente en el final de la vida resulta un tema crítico y frecuentemente controversial. Esta representa un desafío ético-legal para el paciente, sus familiares y el equipo médico ${ }^{(1)}$. Es por esto por lo que decidimos enfocarnos en la nutrición en el final de la vida, para puntualmente generar una discusión y controversia a partir de conceptos teóricos y prácticos, desde la perspectiva de un servicio quirúrgico oncológico, como lo es el nuestro, en donde manejamos pacientes con cáncer de hígado, vías biliares y de páncreas.

El objetivo principal de este artículo es revisar los aspectos teóricos establecidos en la literatura sobre la definición de final de vida, los factores que afectan la calidad en esta etapa de vida y la variedad de estrategias nutricionales disponibles hoy en día para estos pacientes. Finalmente, plantearemos algunos puntos que, con base en la experiencia y juicio clínico y personal, podrían ser una alternativa en el enfoque nutricional del paciente en estas condiciones, que lejos de ser ideas pretenciosas, solo buscan plantear el resultado de un ejercicio quirúrgico y médico juicioso en pacientes con cáncer hepatobiliar y pancreático en el final de vida.

\section{CUIDADOS PALIATIVOS Y LOS CUIDADOS AL FINAL DE VIDA}

La Organización Mundial de la Salud (OMS) define los cuidados paliativos como una intervención que mejora la calidad de vida de los pacientes y sus familias, que enfrentan problemas asociados con enfermedades que amenazan la vida, a través de la prevención y el alivio del sufrimiento, mediados por una identificación temprana y tratamiento del dolor y de otros problemas, tanto físicos, como psicosociales y espirituales ${ }^{(2)}$. Por otro lado los cuidados al final de la vida se centran específicamente en el cuidado de las personas que están cerca del final de la vida, con el fin de permitirles una muerte digna. Aunque es difícil de predecir, el cuidado al final de la vida generalmente se restringe a los pacientes que se encuentran en el último año de la vida, aunque de acuerdo con la legislación de cada país, usualmente este se centra en los últimos 6 meses de vida ${ }^{(3)}$.

En Colombia, el Ministerio de Salud define la enfermedad en fase avanzada o terminal como una enfermedad médicamente comprobada, avanzada, progresiva e incontrolable, que se caracteriza por la ausencia de posibilidades razonables de respuesta al tratamiento, por la generación de sufrimiento físico-psíquico, a pesar de haber recibido el mejor tratamiento disponible, y cuyo pronóstico de vida es inferior a 6 meses ${ }^{(3)}$.

\section{FACTORES QUE AFECTAN LA CALIDAD DE VIDA EN EL PACIENTE EN EL FINAL DE LA VIDA}

Cuando un paciente se encuentra en el final de la vida y los tratamientos curativos no son viables, el enfoque del manejo cambia radicalmente hacia la búsqueda de la calidad de vida ${ }^{(4,5)}$. Por tanto, es importante entender cuáles son los factores que afectan la calidad de vida y la muerte en estos pacientes, teniendo en cuenta la subjetividad que acarrea la interpretación de estos. Existen múltiples factores que influencian su entendimiento, esto incluye la cultura, el tipo y el estadio de la enfermedad, así como el rol social y profesional del paciente en la experiencia de la muerte ${ }^{(6)}$. Sin embargo, en los últimos 20 años, la literatura que trata este tema ha venido creciendo. El estudio Coping with Cancer, por ejemplo, es un estudio longitudinal que evaluó durante 6 años, 
la calidad de vida de los pacientes y la angustia de sus cuidadores en la última semana de vida ${ }^{(7)}$.

Dentro de los principales factores asociados con una mejor calidad de vida se encontraron menor cantidad de estancia en unidades de cuidados intensivos (UCI), y una mayor utilización de hogares especializados para cuidados paliativos $^{(8,9)}$. Asimismo, distintos estudios han identificado otros factores asociados con un puntaje mayor en las escalas de calidad al final de la vida, como lo son la meditación o pertenecer a una religión, así como aquellos pacientes que presentan características psicosociales, como la ausencia de preocupación y la tranquilidad ${ }^{(10)}$.

Un subanálisis de este estudio en 726 pacientes se centró en identificar los factores asociados con menor calidad de vida en la última semana de vida ${ }^{(7)}$. Se identificaron factores principales que deterioran la calidad de vida, la muerte en un hospital y la estancia en la UCI. Asimismo, se encontró una asociación negativa entre aquellas intervenciones más agresivas, como la quimioterapia y sondas de nutrición, y una peor calidad de vida, lo que sugiere que este tipo de intervenciones se deben evitar ${ }^{(8)}$.

Un estudio australiano del 2008, en el que se entrevistaron dos grupos de profesionales de la salud de dos instituciones diferentes, identificó la angustia de los cuidadores frente a la no provisión de alimentación e hidratación, como un factor importante asociado con la tranquilidad y el cuidado ${ }^{(8)}$. Algunas veces, los familiares solicitan continuar la nutrición, pues temen que su familiar "muera de hambre", y muchas veces asocian al profesional médico como cómplice en la muerte del paciente $^{(1)}$. Por esta razón es importante recalcar la importancia de la relación médico-paciente, enfocada en una respuesta adecuada a las preferencias de tratamiento del paciente y un cuidado que les permita a los pacientes lograr un sentido de realización de la vida ${ }^{(11)}$.

\section{IMPORTANCIA DEL SOPORTE NUTRICIONAL EN EL PACIENTE AL FINAL DE LA VIDA}

En la fase del final de vida, la aparición de caquexia secundaria al cáncer es frecuente. En algunos casos esta se convierte en refractaria, lo que quiere decir que no presenta respuesta ni al tratamiento antitumoral ni al soporte nutricional ${ }^{(12)}$. La caquexia refractaria se define como aquella asociada con la pérdida involuntaria de peso, que incluye la disminución del tejido muscular y pérdida de la funcionalidad, la cual no responde al tratamiento nutricional, y esta relacionada frecuentemente con una expectativa de vida menor de 3 meses $^{(12)}$. En la práctica clínica es fundamental evaluar la condición del paciente, ya que desde un enfoque nutricional, pese al manejo invasivo, en ciertos casos, el pronóstico del paciente no mejorará (la carcinomatosis peritoneal o la ascitis refractaria maligna, entre otros) ${ }^{(12)}$.

Actualmente, el término "final de vida" es ambiguo, y los profesionales de la salud no podemos predecir con certeza el tiempo en el que se evidencie un desenlace mortal (podría corresponder entre días, semanas o meses $)^{(12)}$. Se ha propuesto que el soporte nutricional al final de la vida no proveerá beneficio alguno sobre el estado nutricional real del paciente o el desenlace del mismo. El paciente oncológico en el final de vida requiere una evaluación multidisciplinaria, teniendo en cuenta tanto el avance de la enfermedad, como la condición irreversible de la misma y la posibilidad de un tiempo máximo de sobrevida de 3 meses $^{(12)}$.

El cuidado paliativo y la nutrición al final de la vida deben ir de la mano, enfocándose más en la calidad de vida y comodidad del paciente, que en mantener el estado físico del paciente y su funcionalidad ${ }^{(12)}$. Es importante puntualizar que la nutrición tiene valor simbólicoemocional tanto para el paciente, como para su familia y cuidadores ${ }^{(12,13)}$. Es importante recalcar que las intervenciones nutricionales, tales como la nutrición parenteral, enteral (sonda nasogástrica, nasoyeyunal, gastrostomía) y los suplementos nutricionales, se implementan bajo indicaciones claras por parte no solo del médico tratante (cirujano hepatobiliar, en estos casos), sino del servicio de nutrición ${ }^{(12)}$. Por lo anterior, tan pronto se establece la nutrición como una intervención médica, se deben evaluar los riesgos y los beneficios para el paciente, contando también con su consentimiento para iniciar o detener dichas intervenciones.

Uno de los objetivos del manejo paliativo radica en mantener una comunicación adecuada entre familiares y el mismo paciente, para evitar las falsas expectativas y el estrés generado por la ausencia de ingesta de alimentos y la pérdida marcada de peso ${ }^{(12,13)}$. Es importante definir que el paciente, en muchos casos, requiere asistencia para la hidratación y alimentación, sin embargo, este no debe ser presionado, en ninguna circunstancia, para la ingesta, debido a que en muchos casos, los sentidos, como el olfato y el gusto, se ven comprometidos, lo que convierte la ingesta oral en una tarea imposible.

Una vez definido que el propósito del soporte nutricional en el paciente al final de la vida es la calidad de vida y la comodidad, se establece que la valoración del estado nutricional pierde completamente su propó- 
sito, así como el consumo de suplementos orales, para los cuales se ha recomendado limitar su uso en estos pacientes. Se debe recalcar que en estos casos, el principio de autonomía del paciente es imperativo sobre las decisiones médicas, por tanto, se puede afirmar que el médico define la indicación, y el paciente determina el inicio de la terapia ${ }^{(12,14,15)}$.

De acuerdo con lo anterior, el manejo nutricional basal de estos pacientes debe enfocarse en lograr los requerimientos diarios para macro y micronutrientes. En caso de no ser factible se debe enfocar en complacer los deseos del paciente, lo que genera comodidad a la hora de la ingesta mediante el empleo de alimentos fácilmente digeribles, y evita las restricciones alimentarias ${ }^{(12)}$.

\section{PERSPECTIVA DESDE EL CUIDADO PALIATIVO}

Anteriormente, el soporte nutricional se consideraba como un "cuidado ordinario", algo "básico" que de ninguna manera podía negarse al paciente ${ }^{(12)}$. Ha sido considerado un acto humanitario con gran significado emocional y simbólico, que involucra las creencias familiares en cuanto a disminución del dolor, mejoría en la efectividad de los tratamientos, restablecimiento de la funcionalidad y mantenimiento de la dignidad del ser querido. De igual manera, las creencias culturales, en donde se asocia la falta de nutrición con muerte acelerada y las creencias del paciente, quien ve la nutrición como símbolo de amor de su familia, son elementos fundamentales para tener en cuenta ${ }^{(15,16)}$. Desde cualquier perspectiva, ya sea paliativista o no, el soporte nutricional es una intervención médica y no únicamente parte del cuidado básico de los pacientes. Como tal, se debe realizar una evaluación objetiva e integral, en donde se cuente con escalas de medición, que brinden herramientas al clínico para realizar una prescripción adecuada, basada en objetivos claros ${ }^{(17)}$.

Debemos también considerar las contraindicaciones para el manejo nutricional invasivo. En primera instancia se encuentra el discernimiento del paciente. El no deseo del paciente de un manejo nutricional invasivo es una de las principales causas para no iniciar estas terapias. Asimismo, dentro de otras contraindicaciones se encuentran las infecciones de tejidos blandos y los síndromes asociados con la nutrición parenteral, como el síndrome de realimentación ${ }^{(17)}$.

En este orden de ideas es importante preguntarse ¿existe una indicación médica para iniciar el soporte nutricional? ¿De qué tipo de soporte se beneficia el paciente? ¿quién se encargará de su administración?
¿Cuál es la funcionalidad del tracto gastrointestinal? ¿Tiene el paciente alguna vía de alimentación instaurada? ¿Cuánto tiempo durará el soporte? Y, finalmente, ¿cuándo se debe suspender? ${ }^{(18)}$.

Desde la perspectiva médica de los cuidados paliativos existen indicaciones claras para establecer el apoyo nutricional, entre las cuales se encuentran una sobrevida mayor de 3 meses, índice de Karnofsky mayor de 50, desnutrición grave o deseo explícito del paciente. De igual manera se consideran contraindicaciones una sobrevida menor de 3 meses, índice de Karnofski menor de 50, Palliative Performance Scale (PPS) mayor de $20^{(17)}$.

Una vez se define si el paciente es candidato o no a terapia nutricional se debe realizar un manejo integral multidisciplinario individualizado, que implica tanto una valoración por el grupo de soporte nutricional, como medidas que provean bienestar, mejoría de la calidad de vida del paciente y tranquilidad a su familia. Ejemplo de estas medidas son el ajuste apropiado de dentaduras postizas y el uso regular de las mismas, detección temprana de lesiones orales, pequeñas cantidades de líquido vía oral, adecuada higiene bucal y saliva artificial para pacientes con xerostomía, que le permitan mejorar la sequedad bucal, la alteración del gusto y las dificultades para masticar ${ }^{(19)}$.

Es importante recalcar que el objetivo principal que plantea los cuidados paliativos con respecto a la nutrición de los pacientes al final de la vida no es corregir la desnutrición, pues esta en definitiva no corresponde a la causa, sino a la consecuencia de la enfermedad. El fin último es tratar de controlar los síntomas y mejorar la calidad de vida del paciente al fomentar espacios en torno a la ingesta para compartir con sus familiares. Asimismo, se debe evitar conductas o intervenciones, como dietas restrictivas o aditamentos molestos que pretendan la recuperación, ya que no consiguen beneficios clínicamente significativos. Como consecuencia se deben individualizar las decisiones médicas, y ponderar el riesgo y los beneficios, los criterios pronósticos y lo consensuado con el paciente y sus familiares, haciendo la salvedad de que si alguna de las intervenciones entra en conflicto con nuestros objetivos de proveer confort al paciente se debe considerar su retiro.

\section{ESTRATEGIAS DE MANEJO NUTRICIONAL}

Dentro del cuidado básico de los pacientes en el final de vida se debe individualizar las necesidades del paciente y las condiciones sociales, creencias religiosas y deseos tanto del paciente como de su entorno familiar. La hidratación y el control del dolor son pilares fundamentales ${ }^{(13)}$. 
Con respecto al uso de los suplementos nutricionales, en el 2006, la National Collaborating Centre for Acute Care definió su beneficio en aquellos pacientes con intención curativa, en los casos en los que no se logre cumplir los requerimientos nutricionales con la ingesta vía oral únicamente y con propósitos de repleción previa a un manejo quirúrgico u oncológico ${ }^{(13)}$. Sin embargo, en los pacientes al final de vida que presenten síntomas gastrointestinales, secundarios a su patología de base y por tratamientos (quirúrgicos, médicos), estos suplementos no parecen tener un beneficio significativo ${ }^{(13)}$. Por otro lado, el uso de la nutrición parenteral o enteral ha sido otra estrategia de manejo nutricional en pacientes con cáncer y con plan de manejo quirúrgico u oncológico. Sin embargo, su beneficio en pacientes al final de la vida no está definido y, por el contrario, establece una enorme controversia ética con respecto a su uso y retiro de los mismos. Adicionalmente, el uso de nutrición parenteral debe ser restringido a aquellos pacientes en donde la función intestinal esté comprometida, o cuando el acceso nutricional intestinal no sea posible y bajo específicas condiciones discutidas en una junta multidisciplinaria, en donde se analicen los beneficios en la calidad de vida y funcionalidad, y se evalúen los riesgos y posibles complicaciones ${ }^{(13)}$.

\section{DISCUSIÓN}

Las definiciones establecidas para el paciente al final de vida, y los diferentes enfoques del manejo nutricional están claramente establecidos; sin embargo, ¿cómo llevar todo esto a la realidad? La patología HPB afecta directamente al tracto gastrointestinal, en donde se representan la gran mayoría de su sintomatología: hiporexia, náuseas, emesis, dolor abdominal, entre otros ${ }^{(20)}$.

Una cantidad significativa de pacientes con cáncer de páncreas, hígado y vías biliares cursan con dificultad para la ingesta, ya sea por las consecuencias esperadas del cáncer o por la obstrucción maligna del estómago, duodeno o yeyuno, entre otros. Se presentan diversas situaciones difíciles de manejar, por un lado, el paciente muestra una pérdida del apetito importante y, por el otro, la familia, en su enorme preocupación por nutrir a su familiar, se encuentra estresada, finalmente el grupo médico no siempre da las respuestas apropiadas, y no se presenta la mejor comunicación entre todos.

Entonces, ¿qué hacer frente a un paciente con cáncer de páncreas o de hígado que, independientemente de su proceso de enfermedad, llega al final de la vida y no puede comer porque presenta carcinomatosis peritoneal y obstrucción intestinal maligna? En las tradicio- nales juntas quirúrgicas se han discutido estos casos, y se plantea la necesidad de nutrir al paciente. La mejor opción se discute en los grupos multidisciplinarios con cirugía, nutrición, cuidados paliativos, gastroenterología y grupo de ética. Algunos autores refieren que se debe llevar a cirugía y realizar una gastrostomía o una yeyunostomía para alimentar al paciente por sonda mientras fallece ${ }^{(22-24)}$.

Otros autores hablan de colocar una sonda nasoyeyunal avanzada, y garantizar algún aporte proteicocalórico; otros mencionan que lo mejor es suspender la nutrición, y que la enfermedad misma siga su historia natural; otros plantean nutrición parenteral mediante un acceso periférico para garantizar un aporte básico o incluso un aporte subcutáneo de glucosa. Por esta razón nos preguntamos, ¿hay lugar para las intervenciones nutricionales en el paciente al final de vida? ${ }^{(5,23)}$.

En el contexto hospitalario, al realizar una asociación entre nutrición y vida, en nuestro caso a las familias, se plantea la idea de ofrecerle alternativas nutricionales a los pacientes, "se va a morir de hambre y no del cáncer", mencionan ellos. En muchos casos, los pacientes se encuentran en condiciones nutricionales y generales muy deterioradas, con alteración en el estado de conciencia, que muchas veces limita su capacidad de toma de decisiones. Entonces, ¿qué se debe hacer? Se plantean las opciones invasivas frente a las no invasivas, aplicar los criterios que favorecen la restricción de las intervenciones médicas frente a los paternalistas, eutanasia frente a no eutanasia.

Para aquellos que debemos enfrentar el dilema de la terapia nutricional en un paciente en el final de vida de forma rutinaria, esto no resulta sencillo. Ver los ojos del paciente que sufre y de la familia que siente el dolor de la impotencia, observar la propia mirada de nosotros, los médicos tratantes, capaces de hacer grandes cirugías para las que hemos dedicado muchos años en perfeccionar, pero que al final no sirven para nada, y estamos de brazos cruzados viendo como la enfermedad y su curso natural siempre prevalecen ${ }^{(5,23)}$.

La evidencia demuestra que la tendencia actual se encuentra dirigida a individualizar cada paciente, su patología de base, su pronóstico médico, la expectativa de vida aproximada según su enfermedad, las creencias religiosas, la condición socioeconómica y, por supuesto, la participación de una junta multidisciplinaria, que permita la discusión desde el bienestar y perspectiva del paciente ${ }^{(23-26)}$.

El aporte nutricional del paciente en el proceso del final de la vida, desde nuestra experiencia de cirugía 
HPB, está encaminada a las necesidades del paciente, a sus gustos, creencias y sus más íntimos deseos. Está claro que, si somos estrictos con la teoría y los conceptos nutricionales, el paciente cuenta con unos requerimientos energéticos basales para suplir sus necesidades básicas. Sin embargo, la manifestación de la resecabilidad y la posibilidad de curar, desde el punto de vista médico, es nuestro aporte $y$, desde esa perspectiva, no tiene sentido el someter al paciente a ningún tipo de procedimiento invasivo, ya sea nutrición parenteral o enteral por sonda nasoyeyunal, gastrostomía o yeyunostomías. Los suplementos nutricionales serían ideales, pero desafortunadamente generan incomodidad por su sabor, no son consumidos por la falta de apetito y, lejos de dar el aporte mínimo, son una falsa idea de pretender aportar a la recuperación nutricional del paciente, y parecen un efecto placebo para la familia en el contexto de una sensación de tranquilidad al ver a su familiar comer.

Creemos en dar gusto al paciente en lo que desee, que la familia entienda con claridad que no hay limitaciones en sus deseos alimenticios en esta etapa, así no cumpla los requerimientos nutricionales. Parece todo esto contradictorio, pero es dejar de ser clínicos, nutricionistas, cirujanos y convertirnos simplemente en seres humanos, que comprenden y entienden la situación del paciente, tratando de mostrar empatía con la situación del paciente, y brindándole el mayor confort posible tanto a él como a su familia.

En resumen, el manejo nutricional del paciente en el final de vida siempre será un enorme reto ético y moral para el médico tratante. No existe evidencia de qué es lo mejor en términos de nutrición en esta etapa. Existen aproximaciones teóricas del deber ser desde la alopatía. Es por esto por lo que planteamos, desde nuestra experiencia en cirugía HPB, con un volumen de más de 200 procedimientos anuales, que menos es más. Nuestra convicción es que se debe permitir que el curso de la enfermedad siga cuando se han agotado las opciones curativas, y entregar el poder de decisión al paciente de lo que desea, sin necesidad de someterlo a procedimientos invasivos de ningún tipo, si así lo desea o si se encuentra indicado. Es por esto por lo que debemos diferenciar qué intervenciones serán útiles y cuales fútiles, y saber identificar adecuadamente el momento por el que atraviesa un paciente, para, de esta forma, evitar el encarnizamiento terapéutico.

La experiencia en la creación y desarrollo del grupo de cirugía HPB del Hospital Méderi Bogotá, Colombia, nos ha brindado las herramientas para asegurar con cer- teza muchas de las ideas acá planteadas. Seguramente afirmar en una publicación de nutrición que la mejor alternativa de apoyo nutricional del paciente al final de la vida es romper los paradigmas aprehendidos, y simplemente aceptar que la naturaleza de la vida tiene final. Cuestionar la utilidad de suplementos nutricionales o de apoyos de otra índole puede ser paradójico y controversial; sin embargo, estamos convencidos que romper el statu quo hace parte de nuestro quehacer diario, siempre en beneficio de los pacientes. No consideramos que el cirujano deba ser esa retórica figura, limitada únicamente al quehacer dentro del quirófano. Por el contrario, debe ser el líder, el médico cercano al acompañante, el consejero y la mano amiga que ofrezca las diferentes alternativas al paciente y a su familia en todos los aspectos médicos que sean necesarios. $\mathrm{Al}$ ser el que mejor conoce la patología está en la obligación y responsabilidad de orientar a todo el grupo tratante, familia y paciente.

El inevitable desenlace del paciente en el final de vida debe comprometer a todo el grupo de trabajo médico a brindar apoyo, acompañamiento y solidaridad. Aceptar la realidad solo significa que hay que dignificar al ser humano médico y paciente, al brindar solo calidad de vida en el proceso de muerte.

\section{CONCLUSIÓN}

El manejo nutricional de los pacientes oncológicos al final de la vida es un enorme reto médico. La experiencia del servicio HPB aporta una perspectiva humana y paliativa, enfocada en el acompañamiento del paciente y su familia, recuperando el concepto fundamental de la relación médico-paciente.

\section{Agradecimientos}

A cada uno de los pacientes HPB del Hospital Méderi en Bogotá, Colombia, quienes han sido nuestra verdadera escuela y de quienes aprendemos todos los días.

\section{Financiamiento}

El presente estudio no tuvo financiación alguna.

\section{Declaración de autoría}

JCSO participó en la coordinación, concepción, realización, edición y aprobación final del manuscrito. CSG participó en la elaboración, búsqueda de la literatura y realización del manuscrito. ACO participó en la elabo- 
ración y realización del manuscrito. CERC participó en la concepción, búsqueda de la literatura y realización del manuscrito. DCM participó en la concepción, búsqueda de la literatura y realización del manuscrito.

Todos los autores aprobaron la revisión final del manuscrito.

\section{Conflicto de intereses}

Ninguno de los autores declara algún conflicto de interés.

\section{Referencias bibliográficas}

1. van der Riet P, Good P, Higgins I, Sneesby L. Palliative care professionals' perceptions of nutrition and hydration at the end of life. Int J Palliat Nurs. 2008;14(3):145-51. doi: 10.12968/ijpn.2008.14.3.28895.

2. Worldwide Palliative Care Alliance, World Health Organization. Global atlas of palliative care at the end of life. 2014.

3. Krau SD. The difference between palliative care and end of life care: more than semantics. Nurs Clin North Am. 2016;51(3):ix-x. doi: 10.1016/j.cnur.2016.07.002.

4. ABECÉ cuidados paliativos [Internet]. Ministerio de Salud. 2018. (Consultado el 15 de octubre de 2020). Disponible en: https:// www.minsalud.gov.co/sites/rid/Lists/BibliotecaDigital/RIDE/ VS/PP/ENT/abece-cuidados-paliativos.pdf

5. Peppercorn JM, Smith TJ, Helft PR, DeBono DJ, Berry SR, Wollins DS, et al. American society of clinical oncology statement: toward individualized care for patients with advanced cancer. J Clin Oncol. 2011;29(6):755-60. doi: 10.1200/ JCO.2010.33.1744.

6. Hales S, Zimmermann C, Rodin G. The quality of dying and death. Arch Intern Med. 2008;168(9):912-8. doi: 10.1001/ archinte.168.9.912.

7. Garrido MM, Prigerson HG. The end-of-life experience: modifiable predictors of caregivers' bereavement adjustment. Cancer. 2014;120(6):918-25. doi: 10.1002/cncr.28495.

8. Zhang B, Nilsson ME, Prigerson HG. Factors important to patients' quality of life at the end of life. Arch Intern Med. 2012;172(15):1133-42. doi: 10.1001/archinternmed.2012.2364.

9. Wright AA, Keating NL, Balboni TA, Matulonis UA, Block SD, Prigerson HG. Place of death: correlations with quality of life of patients with cancer and predictors of bereaved caregivers' mental health. J Clin Oncol. 2010;28(29):4457-64. doi: 10.1200/JCO.2009.26.3863.

10. Balboni T, Balboni M, Paulk ME, Phelps A, Wright A, Peteet J, et al. Support of cancer patients' spiritual needs and associations with medical care costs at the end of life. Cancer. 2011;117(23):5383-91. doi: 10.1002/cncr.26221.

11. Mack JW, Block SD, Nilsson M, Wright A, Trice E, Friedlander $\mathrm{R}$, et al. Measuring therapeutic alliance between oncologists and patients with advanced cancer. Cancer. 2009;115(14):3302-11. doi: https://doi.org/10.1002/cncr.24360.

12. Orrevall Y. Nutritional support at the end of life. Nutrition. 2015;31(4):615-6. doi: 10.1016/j.nut.2014.12.004.

13. Gillespie L, Raftery AM. Nutrition in palliative and end-oflife care. Br J Community Nurs. 2014;S15-20. doi: 10.12968/ bjen.2014.19.Sup7.S15.

14. Mohanti BK. Ethics in palliative care. Indian J Palliat Care. 2009;15(2):89-92. doi: https://doi.org/10.4103/09731075.58450 .

15. Beijer S, Vogel J, Jager-Wittenaar H. Alternative terminology for the confusing term "palliative nutrition". Clin Nutr. 2017;36(6):1723-4. doi: 10.1016/j.clnu.2017.09.012.

16. DeLegge M, McClave S, Disario J, Baskin W, Brown R, Fang $\mathrm{J}$, et al. Ethical and medicolegal aspects of PEG-tube placement and provision of artificial nutrition therapy. Gastrointest Endosc. 2005 62:952-9. doi: 10.1016/j.gie.2005.08.024.

17. Marcolini EG, Putnam AT, Aydin A. History and perspectives on nutrition and hydration at the end of life. Yale Journal of Nutrition and hydration in Palliative Medicine. Gaceta Mex Oncol. 2013;12(4):267-75.

18. Dev R, Dalal S, Bruera E. Is there a role for parenteral nutrition or hydration at the end of life? Curr Opin Support Palliat Care. 2012;6:365-70. Doi: 10.1097/SPC.0b013e328356ab4a.

19. Lembeck ME, Pameijer CR, Westcott AM. The role of intravenous fluids and enteral or parenteral nutrition in patients with life-limiting illness. Med Clin North Am. 2016;100(5):113141. doi: 10.1016/j.mcna.2016.04.019.

20. Perone JA, Riall TS, Olino K. Palliative care for pancreatic and periampullary cancer. Surg Clin North Amer. 2016;96(6):141530. doi: https://doi.org/10.1016/j.suc.2016.07.012.

21. Woodrell CD, Hansen L, Schiano TD, Goldstein NE. Palliative care for people with hepatocellular carcinoma, and specific benefits for older adults. Clin Therapeutics. 2018;40(4):51225. doi: https://doi.org/10.1016/j.clinthera.2018.02.017.

22. Bozzetti F. Is there a place for nutrition in palliative care? Support Care Cancer. 2020;28(9):4069-75. doi: 10.1007/ s00520-020-05505-x.

23. Macken L, Mason L, Evans C, Gage H, Jordan J, Austin M, et al. Palliative long-term abdominal drains versus repeated drainage in individuals with untreatable ascites due to advanced cirrhosis: study protocol for a feasibility randomised controlled trial. 2018;19(1):401. doi: 10.1186/s13063-018-2779-0.

24. Larson AM. Palliative care for patients with end-stage liver disease. Curr Gastroenterol Rep. 2015;17(5):440. doi: 10.1007/s11894-015-0440-6.

25. Barrocas A, Geppert C, Durfee SM, Maillet JO, Monturo C, Mueller C, et al. A.S.P.E.N. ethics position paper. Nutr Clin Pract. 2010;25(6):672-9. doi: 10.1177/0884533610385429.

26. Druml C, Ballmer PE, Druml W, Oehmichen F, Shenkin A, Singer $\mathrm{P}$, et al. ESPEN guideline on ethical aspects of artificial nutrition and hydration. Clin Nutr. 2016;35(3):545-56. 